\title{
A Novel Algorithm for Power Saving and Over Head Reduction in the Wireless Networks
}

\author{
Dr. M.V. Subramanyam ${ }^{1}$, P.V. Gopi Krishna Rao ${ }^{2}$ \\ ${ }^{I}$ Professor of ECE, Rajeev Gandhi Memorial College of Engg. \& Tech, Nandyal, Kurnool Dist, A.P., India \\ ${ }^{2}$ Associate Professor, Rajeev Gandhi Memorial College of Engg. \& Tech., Nandyal, Kurnool Dist, A.P., India
}

\begin{abstract}
The majority of the nodes connected and operating as an today are Wireless networks, because of its own advantage. In the Adhoc networks nodes are not connected with any fixed infrastructure and are operated by battery power. Each node consumes a large amount of energy while transmission or reception of packets, among the nodes. While the nodes depend on each other for efficient transferring of packets, it is a key issue in networks to have efficient methods for forwarding of packets between any given pair of nodes, with minimum power consumption and less number of intermediate nodes. In this study we propose an optimal routing protocol called MARI (Mobile Agent with Routing Intelligence). The MARI Topology proposed for power management is novel and is used for the consumption of minimum power in an wireless network, at each node. The Protocol groups the network into distinct networks with the selection of MARI nodes and Gateways for efficient packet transmission between any member node pair. The operational cycle at each node is classified into four distinct operations, i.e., transmitting, receiving, idle and sleep cycle, in order to achieve efficient power management in a wireless network.
\end{abstract}

Keywords -Adhoc Networks, MARI, Power management, Nodes, Transmission.

\section{Introduction:}

Wireless networking grows rapidly because of the human desires for mobility and for freedom from limitation, i.e., from physical connections to communication networks. A particular kind of wireless network called mobile network is presently under development, which is the subject of this paper. A mobilenetwork is a self-organizing and rapidly deployable network in which neither a wired backbone nor a centralized control exists. The network nodes communicate with one another over scarce wireless channels in a multi-hop fashion $[1,9]$.In this paper, we propose an algorithm for topology management for the wireless networks: A power management algorithm to reduce the consumption of the power of each node in the wireless networks, by the introduction of MARI (Mobile Agents with Routing Intelligence) topology (a topology having MARI nodes) and management.

The absence of a central infrastructure implies that an wireless network does not have an associated fixed topology. Hence, a most important task of an wireless network consisting of geographically dispersed nodes is to determine (in real time) an appropriate topology over which high-level routing protocols can be implemented [11].

\section{MARI Topology Formation and Management}

Minimizing energy consumption is an important as well as a difficult challenge in mobile networking. The requirement of cooperation between power saving [6] and routing protocols is particularly important in the case of multi-hop wireless networks, where nodes must forward packets from one to another $[3,7,8]$.

This paper proposes a novel topology management scheme for wireless networks for power management called, MARI Topology. The nodes in this scheme are classified into three categories based on their power level. They are:

1. MARI nodes: MARI nodes are selected in such a way that they have the maximum power level among their one-hop neighbors and all non-MARI nodes in the one-hope neighborhood are within the transmission range of MARI nodes. These MARI nodes have the routing intelligence i.e. they make decisions related to routing, such as path finding [4]. Every MARI node has a group of member nodes connected to it, usually in its one-hop neighborhood. The responsibility of every MARI node is to make necessary communication with any member node connected to it or with other MARI nodes (through Gateways) within the network, for both transmission and reception of the packets. MARI nodes are selected or formed by a procedure that is explained later in this paper.

2. Gateway nodes: The Gateway nodes having sufficient power level are selected by the MARI nodes such that they can be used to forward packets between MARI nodes [2,12]. Any two adjacent MARI nodes (within two- 
hop distance usually) in the network are connected through the concerned Gateway node only. Gateway nodes do not have routing intelligence. The MARI nodes select these Gateway nodes, according to the procedure outlined later. The MARI and the Gateway nodes stay continuously awake to route the packets of other member nodes.

3. Member nodes: A member node is a non-MARI and non-Gateway node. These are the nodes, which want to communicate with each other [13]. Every member node is connected to one of the MARI nodes (some kind of belonging or bonding) through which it transmits or receives the packets. The member nodes wake up only at certain specified time epochs, and for very short periods, during any given beacon period $T$. When a member node wakes up and if it does not have to transmit or receive data, then it goes to sleep mode again, after a brief period. This is the main principle behind the power-efficient operation of the network. The wake-up time epochs of each member node are determined apriority (pre-determined). In our simulation of the operation of an wireless network, this is accomplished with the help of pseudo-random number generator. Also, these wake-up time epochs of a member node are known to its corresponding MARI node and its one-hop neighbor nodes, through the WAKEUP messages that are exchanged at the beginning of a beacon period. Thus, the member node can remain in power saving sleep mode for most of the time $[5,10]$, especially when it is not actively sending or receiving packets. The packets are routed over the virtual backbone consisting of MARI nodes and Gateway nodes, which are awake continuously. This is the main power-saving advantage of the topology that is suggested and nurtured in this paper.

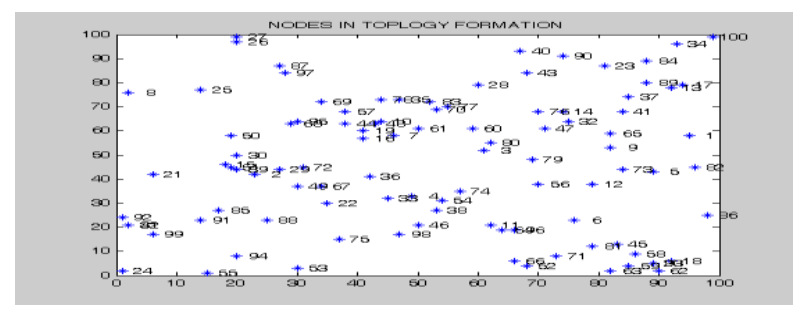

Fig.1 Random distribution of 100 nodes in the wireless network.

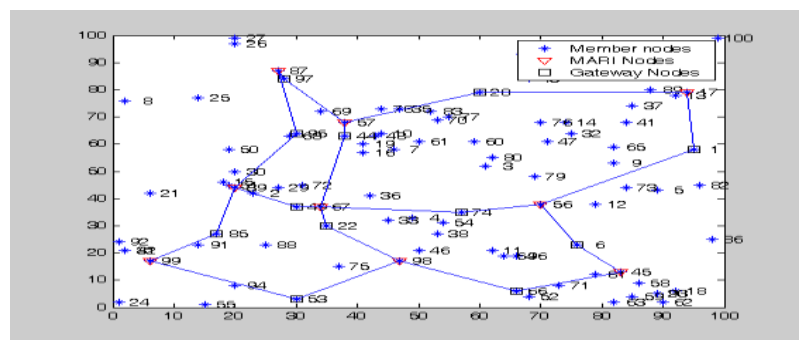

Fig.2 Network with MARI nodes, Gateway nodes and member nodes

The Fig. 2 is the result after executing the following algorithms:

(1) MARI Placement algorithm and

(2) Gateway node selection algorithm

From the fig. 2 it is clear that the number of nodes in the virtual backbone is $20 \%$ to $25 \%$ only. For an example, the list of MARI nodes, Gateway nodes and member nodes for the given MARI Topology network is shown in the Table.1. From the table it is clear that the numbers of MARI nodes are: 09, number of Gateway nodes are: 12 , and the number of member nodes are: 79 .

Table.1 List of MARI nodes, Gateway nodes and member nodes.

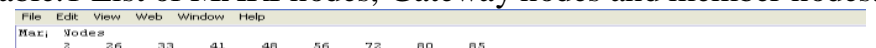

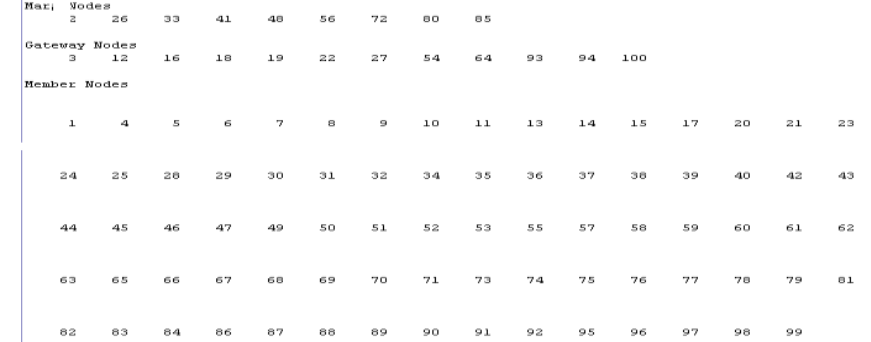

In our simulation, we have considered that the nodes are operating in one of the four modes and their power consumptions are listed in Table-2. 
A Novel Algorithm For Power Saving And Over Head Reduction In The Wireless Networks

Table.2 Power consumption value by each node based on their mode of operation

\begin{tabular}{|l|l|l|l|l|}
\hline Mode & Transmit & Receive & Idle & Sleep \\
\hline Power Consumption & $1400 \mathrm{~mW}$ & $1000 \mathrm{~mW}$ & $830 \mathrm{~mW}$ & $130 \mathrm{~mW}$ \\
\hline
\end{tabular}

III.

Results

We have used MATLAB 7.0 for the simulation of results. In this section we have shown the analysis of wireless network with MARI topology. The results are compared with the existing flat topology. The parameters we considered for analysis and evolution, and their respective results are given below:

a. Overhead in each packet is shown in fig.3.

b. Variation of power at each node in the selected path (given source and destination) is shown in fig.4.

c. Number of packets delivered vs beacon period is shown in fig.5.

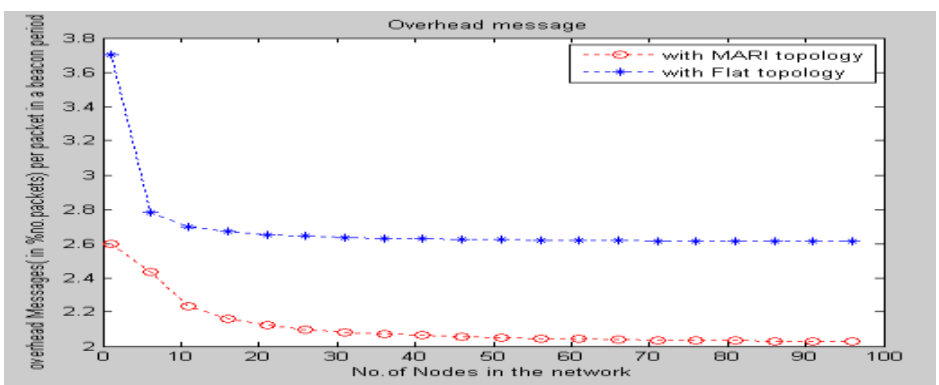

Fig. 3 overhead in each packet per node

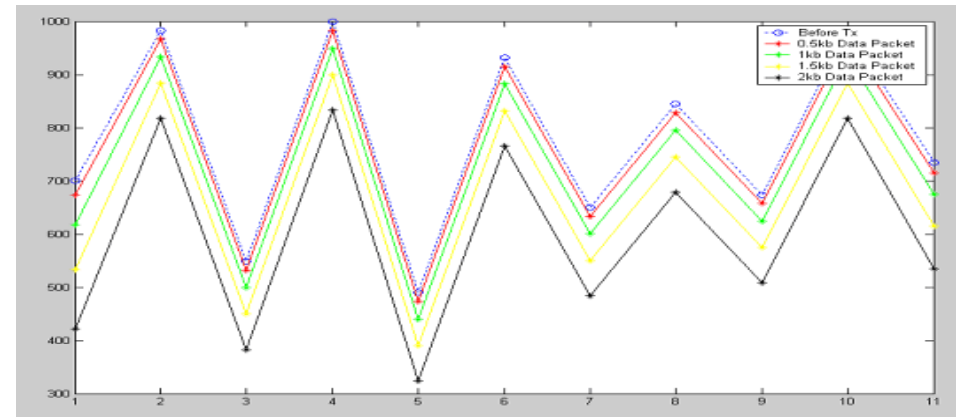

Fig.4 Variation of power at each node in the selected path.

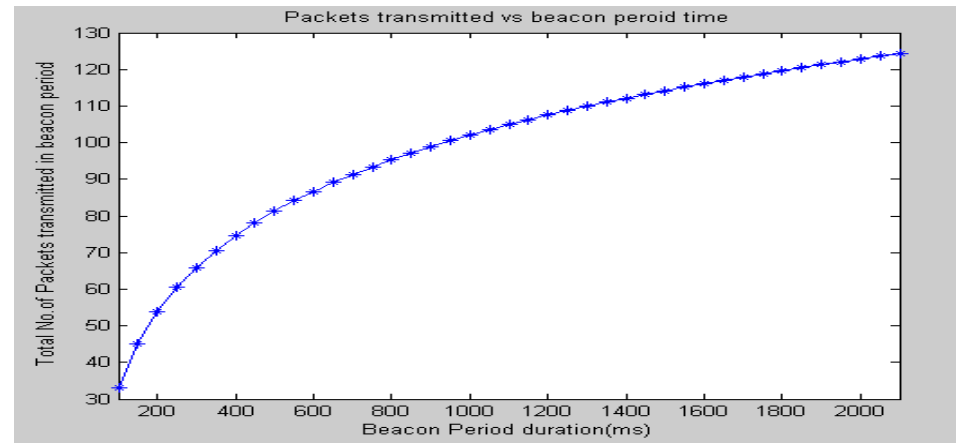

Fig.5 Number of packets delivered as beacon period.

\section{Conclusions}

The performance evaluation of the implemented wireless network is carried out in order (i) to demonstrate the successful operation of the MARI topology concept, (ii) to compare its performance to that of an equivalent (in size) flat-topology network, (iii) to prove the point that the MARI topology performs a way better than an equivalent flat-topology. In our illustration and demonstration, the performance measures that we have considered for simulation study are, (i) the number of backbone nodes as a proportion of the total number of nodes in the network, (ii) packet throughput, (iii) average message delay or turn-around time, (iv) node lifetime, (v) overhead messages, and (vi) average power consumption in the network. Analysis is also done in the following cases:

1. Keeping the data packet size and beacon period constant.

2. Variable data packet size with constant beacon period. 
3. Fixed data packet size with variable beacon period.

We also have shown that the bad effect of misbehavior node in the MARI topology based wireless networks can be overcome by using the protocol called PMP-ANT. A misbehavior node may be a member node or a Gateway node or a MARI node.

\section{Further Scope}

(1) Management protocols for multicasting (one source to Many number of destinations simultaneously) can be designed upon MARI topology or with some further and appropriate modifications to the MARI topology. Load distribution and load balancing among the paths in the network can be considered.

(2) Security levels on each packet, path and node can be implemented, with analysis of security by simulation or bench marking.

\section{References}

[1] Baker, Marti, Giuli, and Lai Baker, "Data/Voice Communication over a Multihop, Mobile, High Frequency Network", proceedings of the IEEEMilitary Communications Conference (MILCOM'97), Nov1997.

[2] Buttyan and Habaux "Packet dropping in the MANET based on their malicious property of the nodes", Proceedings of the 4th ACM International Symposium on Mobile Networking and Computing, (MobiHoc2003), June 2003.

[3] Halpem. J.Y and Li. L "Minimum-energy mo-bile wireless networks revisited," IEEE Inter-national Conference on Communications, June2001.

[4] Hu Y.C., Johnson. D, Jetcheva J.G and Maltz D.A.,"The Dynamic Source Routing Protocol for Mobile Networks", Mobile Ad-hoc Network (MANET) Working Group, IETF, February 2002.

[5] Jung E.-S and Vaidya N. H, "A power control MAC protocol for Ad-hoc networks", ACM MOBICOM, 2002.

[6] Kawadia. V, Kumar P. R., Sreenivas R. S. and Narayanaswamy. S "Power Control in Ad-Hoc Networks: Theory, Architecture, Algorithm and Implementation of the COMPOW Protocol", Proceedings of European Wireless conference, pp. 156-162, Feb. 2002.

[7] Lloyd E.L, Liu.R, Marathe.V, Ramanathan.R and Ravi S. S. "Algorithmic Aspects of Topology Control Problems f o r N e t w o r k s ", Mobihoc, EPFL Lausanne, Switzerland, June 2002.

[8] Meng T. H. and Rodoplu.V, "Minimum energy mobile wireless networks," IEEE Journal on Selected Area on Communications, Vol. 17, No.8,pp 413-418 August 1999.

[9] Perkins C.E "Networking", Addison-Wesley,Boston, 2001

[10] Raghavendra C. S. Singh. S, "Power efficient MAC protocol for multihop radio networks", The Ninth IEEE International Symposium on Personal, Indoor and Mobile Radio Communications, pp. 153- 157, 1998.

[11] Ramanathan. R and Rosales-Hain.R “Topology control of multihop wireless networks using transmit power adjustment”, Proceedings of INFOCOM, pp. 404- 410, 2000.

[12] Sonja .B and Yves Le Boudec. J "Nodes Bearing Grudges: Towards Routing Security, Fairness, and Robustness in Mobile Networks", Proceedings of the Tenth Euro micro Workshop on Parallel, Distributed and Network-based Processing, Canary Islands, Spain, IEEE Computer Society, pp 403-410- January2002.

[13] Sonja .B and Yves Le Boudec.J, "Cooperative routing in mobile networks: Current efforts against malice and selfishness", Proceedings of Mobile Internet Workshop. Informatik 2002- Dortmund, Germany, October 2002. 